\title{
Structuralist approaches to Bohmian mechanics
}

\section{Lorenzo Lorenzetti ${ }^{1}$}

Received: 14 July 2021 / Accepted: 2 November 2021 / Published online: 17 February 2022

() The Author(s) 2022

\begin{abstract}
Lam and Esfeld have argued that, within Bohmian mechanics, the wave function can be interpreted as a physical structure instantiated by the fundamental particles posited by the theory. Further, to characterize the nature of this structure, they appeal to the framework of Ontic Structural Realism (OSR), thereby proposing a structuralist interpretation of Bohmian mechanics. However, I shall point out that OSR denotes a family of distinct views, each of which maintains a different account about the relation between structures and objects, and entails a different kind of ontology. Thus, in this paper I will show how to articulate the structuralist approach to Bohmian Mechanics accordingly to the different standard versions of OSR, and I will evaluate these alternatives. Moreover, I will propose a novel and sui generis kind of structuralist interpretation of Bohmian Mechanics, based on the framework of metaphysical coherentism.
\end{abstract}

Keywords Wave function · Bohmian mechanics · Ontic Structural Realism · Metaphysical coherentism · Primitive ontology · Ontological dependence

\section{Introduction}

Lam (2015) and Esfeld (2017) have argued that, within Bohmian mechanics, the wave function can be interpreted as a physical structure instantiated by the fundamental particles posited by the theory. Furthermore, in order to further characterize the nature of this structure, they appeal to the framework of Ontic Structural Realism (OSR). Broadly speaking, "OSR is any form of structural realism based on an ontological or metaphysical thesis that inflates the ontological priority of structure and relations" (Ladyman 2014). By adopting this strategy, Lam and Esfeld do not simply provide a structuralist interpretation about the ontological status of the wave function alonerather, they are proposing a more general structuralist account of the ontology of

Lorenzo Lorenzetti

lorenzo.lorenzetti@bristol.ac.uk

1 Department of Philosophy, University of Bristol, Cotham House, Bristol BS6 6JL, UK 
Bohmian mechanics, which concerns the nature of both the wave function and the Bohmian particles. ${ }^{1}$ In fact, OSR is a view that provides us with a rich ontological picture that concerns the status of both structures and objects. More precisely, as stated by Ladyman and Ross:

Ontic Structural Realism (OSR) is the view that the world has an objective modal structure that is ontologically fundamental, in the sense of not supervening on the intrinsic properties of a set of individuals. According to OSR, even the identity and individuality of objects depends on the relational structure of the world. (Ladyman et al. 2007, p. 130)

However, it should be pointed out that OSR does not denote a unique view. Rather, it refers to a family of very different positions. Distinct versions of OSR disagree about what kind of entities exist fundamentally and about the exact putative relationship between structures and objects. Furthermore-as I will argue-OSR is not the only framework which is compatible with a structuralist-like interpretation of Bohmian mechanics. Therefore, a lot more can be said, besides the claim that the wave function should be regarded as a structure.

My aim for this paper is twofold. On the one hand, I will confront the main different forms of OSR with Bohmian mechanics. I shall point out that all but one are fundamentally at odds with the intuitive picture suggested by the formalism of Bohmian mechanics. On the other hand, I will propose a novel and sui generis kind of structuralist interpretation of Bohmian mechanics, based on metaphysical coherentism.

The latter account-which has been recently developed by Calosi and Morganti (2021) and Morganti $(2019,2020)$ in opposition to OSR - takes objects as fundamental, provided that an essential part of their qualitative profile stems from 'same-level' symmetric relations of ontological dependence between the objects themselves. This framework provides an object-first ontology, but preserves nonetheless certain structuralist/holistic insights, since it holds that the nature of fundamental objects is not wholly characterizable solely in terms of intrinsic and independent properties. I will argue that we can interpret the wave function in Bohmian mechanics as stemming from the network of dependence relations between the particles postulated by metaphysical coherentism (from now onward, 'coherentism').

The plan for the paper is as follows. In the next section, I will briefly introduce Bohmian mechanics, in order to provide the basic technical information which are needed for the development of the rest of the paper. In the third section, I will introduce the structuralist approach to Bohmian mechanics and discuss how it can be spelt out in terms of the main standard versions of OSR. Finally, in the fourth section I shall put forward and discuss the coherentist account.

\section{Bohmian mechanics and primitive ontology}

Bohmian mechanics is a non-local hidden variable quantum theory whose crucial claim is that particles possess definite positions at all times. According to the standard

\footnotetext{
${ }^{1}$ I am grateful to one anonymous reviewer for helping me to make this point clear.
} 
interpretation of Bohmian mechanics, these particles are objects living in ordinary three-dimensional space. ${ }^{2}$ That is, the three-dimensionally located particles constitute the so-called primitive ontology of the theory. In the words of Dürr et al. $(2012,29)$, "the basic kind of entities that are to be the building blocks of everything else (except, of course, the wave function) [are] particles, described by their positions in space, changing with time.".

Bohmian mechanics describes quantum systems via the standard wave function $\psi=\psi\left(q_{1}, \ldots, q_{n}\right)$, for an $N$-particle system, and-differently from orthodox quantum mechanics - specifies the actual positions $Q_{1}, \ldots, Q_{n}$ of the particles that define the configuration $Q .{ }^{3}$ Furthermore, alongside the standard Schrödinger equation describing the evolution of the wave function, Bohmian mechanics assumes also a guidance equation, which is an equation about the motion of the particles. The two fundamental equations of the theory can be stated as follows: ${ }^{4}$

(1) Schrödinger equation: $i \hbar \frac{\partial \psi}{\partial t}=\hat{H} \psi$

(2) Guidance equation: $\frac{d Q_{k}}{d t}=\frac{\hbar}{m_{k}} \operatorname{Im}\left\{\frac{\psi^{*} \nabla_{k} \psi}{\psi^{*} \psi}\left(Q_{1}, \ldots, Q_{n}\right)\right\}$

Here $Q_{k}$ and $m_{k}$ denote respectively the position and the mass of the $k$-th particle, $\hbar$ is Planck's constant, $\hat{H}$ is the Schrödinger Hamiltonian. Thus, we can say that within this quantum theory the wave function fixes - through the guidance equation above - the temporal evolution of the Bohmian particles. ${ }^{5}$

Three points should be noticed. First, the fundamental wave function for Bohmian mechanics is the universal wave function, i.e. the wave function of the universe, that is the biggest $N$-particles system. Secondly, Bohmian mechanics is non-local, thanks to the fact that - due to the guidance equation - the velocity of each particle at a time $t$ depends on the position of each other particle in the configuration at that same time $t .{ }^{6}$ Finally, concerning the relation between the wave function and the primitive ontology within Bohmian mechanics, we can say that the theory is fundamentally about the behaviour of particles. As Goldstein (2017, pp. 13-14, italics added) claims, "in this sense, for Bohmian mechanics the particles are primary, or primitive, while the wave function is secondary, or derivative". ${ }^{7}$

That being said, Bohmian mechanics raises some important questions. First of all, what does the wave function, and in particular the universal wave function, represent in the world? How is it metaphysically related with the primitive ontology of the theory, besides what we have said so far? That is, what is the fundamental ontology of the world according to Bohmian mechanics? Should it include both the particles and the wave function? Several possible answers are available to these questions.

\footnotetext{
2 Alternatively, Bohmian mechanics can be interpreted as postulating a unique particle living in a $3 \mathrm{~N}-$ dimensional space, taking the wave function as a field defined over that high-dimensional space (Albert 1996). For the purpose of this paper, I shall focus only on the 'three-dimensionalist' view.

3 Here I follow Goldstein and Zanghì (2013) and I use $q_{1}, \ldots, q_{n}$ for generic position and configuration variables and $Q_{1}, \ldots, Q_{n}$ for the actual positions.

4 Cf. Goldstein and Zanghì (2013).

5 See e.g. Lam (2015, p. 86).

6 See Tumulka (2018, p. 8).

7 The same position is shared also by Allori et al. (2008, p. 356).
} 
That is, several different metaphysical accounts about the nature of the wave function in Bohmian mechanics are viable. In this paper I will focus on the structuralist stance.

\section{The OSR interpretation of Bohmian mechanics}

\subsection{Lam and Esfeld's structuralist proposal}

Several accounts have been proposed concerning the nature of the universal wave function. ${ }^{8}$ For example, some have proposed to treat it as a law of nature (e.g. Goldstein and Zanghì 2013), while others have interpreted it as denoting a dispositional property (e.g. Esfeld et al. 2013; Suárez 2015). Here I will focus on another position, namely the structuralist view, which has been recently sketched by Lam (2015) and Esfeld (2017).

This account takes the wave function as corresponding to a network of relations instantiated by the entities belonging to the primitive ontology in three-dimensional space. According to Lam and Esfeld, we should interpret this structure in terms of OSR. ${ }^{9}$ Thus, the structure at stake is said to be metaphysically fundamental, and the relata are somehow dependent on it. More precisely, their central claim is the following:

The wave function is understood in terms of a concrete, physical structure instantiated by the Bohmian particles. [...] For each Bohmian particle, the fact of being this very particle, which includes its own trajectory and dynamical features, depends on the structure it is part of. (Lam 2015, pp. 88-89)

What they argue is that the wave function is a physical structure that relates the position of all the particles, in such a way that "the temporal development of the position of each particle (i.e. its velocity) depends on the positions of all the other particles" (Esfeld 2017, p. 2337). Notice that, according to this proposal, the structure exists over and above the elements of the primitive ontology. That is, the relations which connect all the Bohmian particles are not supervenient on the intrinsic properties of those particles. On the contrary, they are fundamental, and the qualitative profile of the particles depends on the structure they belong to. In this way, as mentioned at the beginning, Lam and Esfeld-by providing an OSR interpretation about the nature of the wave function in this quantum theory—are sketching a structuralist account about the whole ontology of Bohmian mechanics.

Esfeld and Lam do not say much more about the nature of the structure invoked. However, there is more to be said, since this notion of structure and its relationship with the particles can be spelt out in different and more precise ways. Do structures and objects both exist? Are they both fundamental? How are they related precisely? Different structuralist accounts—and, especially, different versions of OSR—entail

\footnotetext{
8 See Belot (2012), Ney and Albert (2013) and Chen (2019) for a complete overview.

9 See for example (Ladyman et al. 2007) and (French 2014). Actually, if we consider the general philosophical framework underlying OSR, as it can be found e.g. in Ladyman et al. (2007), it is clear that OSR is strictly connected with a strong empiricist stance, which could be said to be conflicting with the project of Bohmian mechanics. However, I am focusing here just on the strictly metaphysical aspects of OSR, as Lam and Esfeld_-and a large part of the literature — seem to do.
} 
different ontologies. Therefore, it is necessary to specify in which way the structure invoked by Lam and Esfeld should be interpreted if we want to understand which metaphysical picture of the world is implied by their view.

To be fair, Esfeld (2017, p. 2338) himself gives some suggestions about how one can interpret in a more precise way his structuralist proposal, but I think they are unconvincing. He suggests that we can take this structure to be either a primitive law of nature instantiated by the universe, or a holistic dispositional property instantiated by the totality of the particles-following the account developed in Esfeld et al. (2013). As they claim, "On this view, the universal wave-function, $\Psi_{t}$, of the system of particles at a given time is a mathematical object that represents the disposition to move in a certain manner at that time." (Esfeld et al. 2013, p. 13). However, both these accounts seem to conflict with the proposal according to which the wave function is a (physical) structure. On the one hand, the nomological view interprets it as a law of nature, while on the other hand the dispositional account interprets it as an intrinsic property. Arguably, neither of these two kinds of entities fall under the category of 'structures', nor are they ascribable to the forms of OSR that are discussed in the literature. Therefore, since Esfeld and Lam appeal explicitly to the framework of OSR - and given that this is arguably a distinct framework from the dispositionalist and the nomological ones mentioned above, which should be taken as alternative possible accounts-I will try to spell out their view in standard OSR terms. That is, before conflating the structuralist view with the dispositionalist or nomological accounts, let's see if there are other ways-closer to the structuralist framework as it is discussed in the philosophy of science-to account for the nature of the wave function as a structure.

\subsection{The OSR interpretation made precise}

Given that Esfeld and Lam describe their account as a form of OSR, we can begin by pointing out that OSR is not a single view, but comprises a family of different positions. In the following, I will consider the main forms of OSR and check how they combine with the structuralist interpretation of Bohmian mechanics which Esfeld and Lam have put forward. Thus, I shall focus on eliminativist, priority-based and moderate OSR. All these three versions of OSR are suitable forms of OSR, but they entail different ontologies. This means that Lam and Esfeld's structuralist proposalas I have presented it-cannot give univocal answers to the questions which I have raised at the end of the last section. Thus, it is incomplete. Structuralism cannot be considered as an adequate metaphysical account of Bohmian mechanics until it tells us what is the fundamental ontology of the theory. Therefore, we have first to set out which version of OSR best fits Bohmian mechanics.

Moreover, as I said, Lam and Esfeld define the wave function as a structure in OSR terms. However, I shall argue that the structuralist interpretation can be formulated also via a different framework, i.e. coherentism. This theory embeds a different ontology from every one of the three OSR accounts aforementioned. I shall suggest that the coherentist proposal preserves some of the basic intuitions of the structuralist view, but it displays some advantages over the OSR-formulations of the theory. 
This section is devoted to OSR. Something which is shared by all the three standard versions of OSR is the claim that structural elements are amongst the most fundamental entities composing reality. Moreover, all the forms of OSR support the claim that the objects existing at the fundamental level (if they exist at all) depend-in some sense to be specified-on the structure they belong to.

Let's start by considering eliminativist OSR. Roughly speaking, according to this theory structures are all there is to the world. ${ }^{10}$ Thus, one can combine this view with Bohmian mechanics by eliminating the Bohmian particles from the ontology of the theory. However, it seems that we cannot take seriously this option in our context. In fact, this would amount to the elimination of the primitive ontology. But, on the contrary, Lam and Esfeld are explicitly committed to the existence of Bohmian particles in three-dimensional space. Thus, it seems clear that this version of OSR is not the kind of OSR account they are thinking about, and we can set this aside right away. Therefore, we should rather focus on priority-based or moderate OSR, which grant at least some minimal ontological status to particles. Indeed, these two latter theories posit both particles and structures, and disagree only on the direction and the kind of dependence ${ }^{11}$ relation between the two categories of entities.

Consider firstly priority-based OSR. Roughly speaking, according to this thesis structures are ontologically prior to (or more fundamental than) objects. Nonetheless, both categories of entities are considered equally existent. In the recent literature, this kind of dependence has been formulated in terms of grounding (McKenzie 2017, 2020). For our purpose it will be enough to characterize grounding in the following way. One entity $x$ can be said to ground another entity $y$ only if $x$ metaphysically determines $y$-that is, only if the obtaining of $x$ constitutes a sufficient condition for the existence of $y^{12}$ (Fine 2015). As McKenzie claims, only if we understand priority in terms of determination - and thus in terms of sufficient conditions - then "a full understanding of the fundamental would be, at least in principle, enough to generate an understanding of the world in its entirety". ${ }^{13}$ That is, only within a picture of the fundamental as what determines what is secondary, we can make sense of the metaphor that the fundamental is the least that God would need to build the whole reality. This is the sense in which structures are taken as the most fundamental entities and objects are considered to be ontologically derivative.

To put this more rigorously, let's first formalize grounding in some general terms. Here, I adopt Fine's (2015) notation:

$$
\varphi_{1}, \varphi_{2}, \ldots \rightarrow \psi
$$

(it is the case that $\psi$ in virtue of it being the case that $\varphi_{1}, \varphi_{2}, \ldots$; where $\psi, \varphi_{1}, \varphi_{2}, \ldots$ are open sentences). So, I propose to formulate priority-based structuralism (in the case of two objects determined by a single relation) as follows:

\footnotetext{
10 This is roughly the position defended by French (2014). Ladyman has endorsed a version of this account in the past, even though he has developed a more sophisticated account in the last years (see Ladyman et al. 2007; Ladyman 2015, 2016), which I will not consider further for the purpose of the present paper.

11 I use the term 'dependence' to designate a generic relation. In the following, I shall present two different ways in which dependence can be formulated, i.e. grounding and ontological dependence.

12 This is the metaphysical (i.e. non-causal) counterpart of causal determination.

13 McKenzie (2020, p. 507).
} 


$$
R x_{1} x_{2} \rightarrow\left(x_{1} \wedge x_{2}\right)
$$

(it is the case that $x_{1} \wedge x_{2}$ in virtue of it being the case that $R x_{1} x_{2}$ ).

Thus, if we combine this framework with Lam and Esfeld's proposal, we should say that in the case of Bohmian mechanics the particles are derivative in nature and they are metaphysically determined by the physical structure representing the wave function. However, we can already notice that-just like eliminativism - this option seems to be quite revisionary. As we have seen in the second section, while formulating Bohmian mechanics we start from particles and their positions (i.e. the particle configuration is postulated from the beginning), while the wave function is secondary and specifies the evolution of the physical system. An asymmetric relation of determination going from structures to objects, instead, supports the idea that the wave function is the most fundamental entity. That is, all that has to be posited to construct the whole reality. This is not a decisive argument against this option, of course, but I believe that we should resort to revisionary interpretations only if no other metaphysical account is really viable. ${ }^{14}$

Moving on to the third standard version of OSR, I shall now consider moderate OSR (Esfeld and Lam 2008). We have seen that within priority-based OSR objects are said to be asymmetrically determined by structures. Thus, only structures are fundamental. On the other hand, according to moderate OSR, structures and objects are both fundamental and symmetrically inter-dependent. How should we formulate this notion of inter-dependence? Should we resort to grounding as with priority-based OSR? Since metaphysical determination is meant to express relations of priority, grounding seems to be unsuitable to characterize moderate OSR.

Indeed, moderate OSR has been usually defined via the notion of ontological dependence (e.g. French 2010). The notion of ontological dependence at stake is the one developed by Fine (1995). We can define ontological dependence in the following way, in terms of essence, or identity: " $x$ [ontologically] depends on $y$ just in case part of what it is to be $x$ involves $y$ " (Barnes 2018, p. 52). This concept of dependence is different from the one associated with grounding. In fact, while we can take $y$ to ground $x$ only if $y$ is metaphysically sufficient for $x$, we should take $x$ to be ontologically dependent on $y$ only if $y$ is metaphysically necessary for $x$ (Fine 2015). ${ }^{15}$ Thus, moderate OSR is the thesis that objects are ontologically dependent on structures and vice versa. Each category is necessary for the other one. In French's words:

The identity of the putative objects is (symmetrically) dependent on that of the relations of the structure and vice versa. (French 2010, p. 104)

We can express this relation formally, using Fine's (2015) formulation of ontological dependence:

$$
\varphi \leftarrow{ }_{x, y, \ldots} \psi_{1}, \psi_{2}, \ldots
$$

(it is essential to $x, y, \ldots$ satisfying $\psi_{1}, \psi_{2}, \ldots$ that $\varphi$ be the case; where $\psi, \varphi_{1}, \varphi_{2}, \ldots$ are open sentences and $x, y, \ldots$ are variables). Thus, we can express the relations of

\footnotetext{
14 See also Morganti (2020) on this point.

15 As before-this can be taken as the metaphysical (i.e. non-causal) counterpart of causal dependence.
} 
ontological dependence within moderate OSR in the following way (in the case of two objects symmetrically dependent with a relation $R$ ):

$$
\left(R x_{1} x_{2} \leftarrow x_{1}, x_{2} x_{1}, x_{2}\right) \wedge\left(\left(x_{1} \wedge x_{2}\right) \leftarrow_{R} R x_{1} x_{2}\right)
$$

(it is essential to $x_{1}, x_{2}$ that $R x_{1} x_{2}$ is the case, and vice versa). ${ }^{16}$

Within Esfeld and Lam's structuralist interpretation of Bohmian mechanics, moderate OSR would amount to the thesis that the wave function (as a physical structure) and the spatially located particles are inter-dependent. At least prima facie, this account seems to be slightly less revisionary than the priority-based one. In fact, it grants the existence of particles and is not committed to the claim that the particles are entirely derivative on the wave function.

Summing up, so far we can formulate the ontology of the Bohmian structuralist account in three ways: (i) according to the eliminativist position, the wave function is all there is; (ii) priority-based OSR claims that the Bohmian particles are determined by the wave function; and finally, (iii) according to moderate OSR the wave function and the particles are both fundamental and they are inter-dependent. All that I want to point out here is that-even though all these positions are, strictly speaking, consistentonly the last one seems to fit nicely with the presentation of Bohmian mechanics which I have given in the second section.

Putting OSR aside, in the rest of the paper I shall propose a fourth alternative structuralist account about the ontology of Bohmian mechanics, based on the framework of metaphysical coherentism. I will suggest that this is the most natural way in which a structuralist account of Bohmian mechanics can be formulated.

\section{A coherentist proposal for bohmian mechanics}

Calosi and Morganti (2021) and Morganti (2019, 2020) have recently developed a novel account concerning the metaphysics of quantum entanglement, which they have called metaphysical coherentism. It is a widespread opinion that the state of (say) two entangled subsystems of the world-e.g. a singlet state like: $|\psi\rangle=|\uparrow\rangle_{1}|\downarrow\rangle_{2}-\mid \downarrow$ \rangle$_{1}|\uparrow\rangle_{2}$ - cannot be understood as the conjunction of the subsystem states. Thus, there is more to entangled systems than the particles that constitute them. One strategy to understand the nature of entangled states is to appeal to the OSR framework. The OSRist would claim that entanglement relations are physical relations, and that either the particles which instantiate them are metaphysically determined by them, or that the particles and the entanglement relations are mutually inter-dependent.

On the other hand, Calosi and Morganti (2021) have proposed another alternative, i.e. metaphysical coherentism. My aim for this section is to apply their account to the debate concerning the wave function in Bohmian mechanics. Thus, I shall start by considering Calosi and Morganti's account.

According to them, objects should be regarded as the only fundamental category of entities, but we should also maintain that an essential part of their qualitative

\footnotetext{
16 Notice that, to express the dependence of the relation on the objects (i.e. the second conjunct), we need to use a second-order index $\mathrm{R}$ in place of a first-order index. See Fine $(2015, \mathrm{ft} .4)$ on this issue.
} 
profiles stems from 'horizontal' 17 mutual relations of ontological dependence with other objects. Thus, the peculiar nature of entangled states is "to be interpreted in terms of symmetric relations of ontological dependence between certain groups of non-relational entities" (Calosi and Morganti 2021, p. 33), i.e. the particles.

Like moderate OSR, coherentism is expressed in terms of ontological dependence. However, notice that in the case of OSR the relevant relations of dependence were instantiated between objects and structures. On the other hand, according to coherentism we should regard objects to be dependent on entities of the same kind. When two objects are mutually dependent, the qualitative profile of one object (at least partially) depends on the qualitative profile of the other object, and vice versa, and we cannot wholly characterize the identity of one entity independently from the other. In this way, coherentism can account for the holistic nature of entangled states. Once we take entangled states as characterized by symmetric relations of dependence, since entanglement is a really widespread phenomenon, we end up with what Calosi and Morganti call horizontal webs of dependence. ${ }^{18}$

In the end, this resembles the peculiar structuralist account proposed by Linnebo (2008) in the philosophy of mathematics, which is characterized by the following principle:

$(O D O)$ Each object in $D$ depends on every other object in $D$ (where $D$ is a certain domain).

Just as we did with priority-based and moderate OSR, we can formulate coherentism in a formal way employing Fine's notation. In particular, we can formally represent mutual dependence relations as follows (in the case of two symmetrically dependent objects $x_{1}$ and $x_{2}$ ), in terms of ontological dependence:

$$
\left(x_{1} \leftarrow_{x_{2}} x_{2}\right) \wedge\left(x_{2} \leftarrow_{x_{1}} x_{1}\right)
$$

(it is essential to $x_{2}$ that $x_{1}$ is the case, and it is essential to $x_{1}$ that $x_{2}$ is the case). ${ }^{19}$

I argue that a similar picture can be employed to explain the mutual dependence of each particle's velocity on the other particles' positions in the Bohmian picture. Consider the characterization put forward by the OSRists. For them, the non-local entity represented by the universal wave function is identified with a fundamental physical structure that relates all the particles, which in turn inherit their identity from their place within the structure. On the contrary, according to the coherentist framework one should consider the Bohmian particles as the most fundamental entities, while maintaining that they ontologically depend on each other-at least concerning their particular positions. ${ }^{20}$

\footnotetext{
17 In opposition to the 'vertical' asymmetric relations of dependence which characterize priority-based OSR.

18 From this the name 'coherentism', which is a reference to coherentism in epistemology.

19 Cf. Calosi and Morganti (2021, p. 35). As they point out, it is not simply the existence of (say) $x_{2}$ that is essential to $x_{1}$, but the fact that it exists as the very entity it is.

20 It could be argued that a position like this - i.e. a Bohmian version of the coherentist view-is not merely an instance of the coherentist framework proposed by Morganti and Calosi, but a refined version of their account which is actually necessary to make it consistent. Indeed, the coherentist approach to quantum entanglement, and thus to quantum mechanics, is based on the claim that we can postulate particles as
} 
Let's see how we could express more precisely this notion of ontological dependence. Remember once again that in Bohmian mechanics 'the velocity of each particle at a time $t$ depends on the position of each other particle in the configuration at that same time $t^{\prime}$. As it is rightly pointed out by Lam (2015), there is a dynamical interdependence between the relata. This is because at each instant $t$ it is the velocity of one particle that depends on the position of the other particles at that same instant. Thus, its position depends only "indirectly" on the positions of the other elements of the primitive ontology.

My proposal for a coherentist version of Bohmian mechanics is roughly the following: 21

At each time $t$, each particle ontologically depends, with respect to its position $Q_{k}$ in the configuration, on the velocity of every other particle in the configuration $Q$ at the same time $t$, in the way formally described by the guidance equation.

Summing up, the fact of being that very particle-i.e. the fact of possessing that specific position value-depends, in the appropriate situation, on the determinate position properties of all the other particles, in the way formally described by the mathematical apparatus of Bohmian mechanics, in particular by the guidance equation.

At this point, we can regard the universal wave function as a 'whole' stemming from the horizontal web of dependence relations between the particles that coherentism postulates. At the formal level, the wave function encodes the totality of those dependence relations. It should be stressed that the wave function is not, in this view, a fundamental physical structure on which the Bohmian particles are dependent on. Rather, it represents the dependence relations themselves-holding among the particles. Thus, strictly speaking, the wave function is not a real fundamental ontological posit of the theory, even though it tracks objective features of the world. In this sense, within the coherentist framework, the wave function does not seem to have the same ontological significance that it has in the OSR views. ${ }^{22}$

Indeed, one putative difference between coherentism and OSR concerns the status of the relations which connect the Bohmian particles. On the one hand, according to the

\section{Footnote 20 Continued}

self-substantive (although interdependent) objects, endowed with intrinsic features. Thus, it seems to imply the existence of a primitive ontology that can instantiate the relations of ontological dependence. On the contrary, if one were to assume that the wave function is all there is, then it would be unclear what are the objects which are supposed to be the fundamental posit of the theory. Because of this, I believe that Bohmian mechanics - as presented here-is particularly well suited to accommodate coherentism, and it seems sensible to claim that coherentism actually needs a theory like this (i.e. Bohmian mechanics with a primitive ontology) to be consistent. Another option, for instance, could be to develop the coherentist account in the context of the mass-density version of GRW theory (cf. Allori et al. 2008). Thanks to an anonymous reviewer for pointing this out.

21 I'm considering non-relativistic quantum mechanics here.

22 This renders the coherentist interpretation less 'realist' about the ontological status of the wave function than the OSR interpretation. However, as a metaphysical framework, it can still be deemed as structuralist in flavour due to the modal interdependence which takes place between the objects (which resembles Linnebo's mathematical structuralist account expressed in (ODO)). Thus, one could still take the coherentist interpretation as a refinement of Esfeld and Lam's initial proposal. 
OSR interpretation, the wave function represents a physical structure. On the other hand, coherentism links the particles via ontological dependence relations between them, and Morganti stresses that the latter are not on the same ontological footing of the OSR relations:

Were the coherentist to postulate objects and physical relations, his/her position would be indistinguishable, say, from the moderate OSRists'. The emphasis on ontological dependence entails instead that the basic ontological inventory may well include only objects, even though the whole story concerning their properties will not be told unless it will include some sort of mutual interrelations. The relevant relations will of course be physical in the sense that they are captured and described by physics in some way and at some level, but not in the sense of being as concrete as objects. (Morganti 2019, 18n)

If he is right, then one could maintain that coherentism is more ontologically parsimonious than moderate OSR (but still on the same level as priority-based OSR, which posits only structures at the most fundamental level). Whereas moderate OSR posits both structure and objects, coherentism comprises only the latter within its fundamental ontology. Note that this point relies on the assumption that relations of ontological dependence are really substantially different from the 'physical' relations of OSR. Admittedly, even though there is a prima facie difference between the two, this can be a controversial point. I shall not assess this here, since it regards the broader coherentist framework and it does not fall within the scope of the present discussion, but I would like to suggest that the proponents of metaphysical coherentism should address this issue carefully in the future.

Setting aside the latter issue-and to better clarify the nature of the wave function within the coherentist proposal-it is worth stressing how the coherentist version of Bohmian mechanics can actually be set apart from other interpretative options which can be said to bear some similarities with it, to make clear that the coherentist account can stand alone as a novel proposal, and does not collapse onto other accounts. ${ }^{23}$

Firstly, I want to focus on the Humean account of Bohmian mechanics that has been recently discussed in the literature (see e.g. Miller 2014; Esfeld et al. 2013). ${ }^{24}$ That account takes the lead from David Lewis's doctrine of Humean supervenience, according to which the world is a "vast mosaic of local matters of particular fact" (Lewis 1986, pp. ix-x). Roughly, what exists fundamentally is just an arrangement of points instantiating intrinsic properties and related by spatiotemporal relations, and everything supervenes on that. Applied to the case of Bohmian mechanics, the Humean view claims that all that exists fundamentally is a spatiotemporal distribution of Bohmian particles. Then, according to the Humean approach, the following can be argued:

The universal wave-function and the laws of quantum mechanics supervene on this distribution [i.e. the distribution of the elements in the primitive ontology]. They are nothing more than devices of economical bookkeeping, there being no

\footnotetext{
23 Thanks to an anonymous reviewer for pressing me on this point.

24 See Dewar (2020) for a critical review.
} 
real connections among the elements of the primitive ontology (such as a real relation of entanglement); there is only a non-dynamical background structure of space-time geometry into which these elements are inserted. (Esfeld et al. 2013, p. 792)

Similarly to the coherentist proposal, within this account the wave function is conceived as a descriptive device. However, the two approaches are based on very different metaphysical frameworks. ${ }^{25}$ In fact, within the coherentist framework, the wave function stems from a network of modal relations-i.e. relations of ontological dependence-between the particles. Therefore, the ontology underlying the coherentist account is modally loaded in a way in which the Humean version could not be. In fact, Humeanism is - by definition - an approach that rules out necessary connections (and, more generally, irreducibly modal connections) between entities. On the contrary, coherentism posits non-supervenient modal connections in the world connecting our objects. This fact characterizes coherentism as a form of structuralist ontology and distinguishes it from the Humean account. ${ }^{26}$

Secondly, it is worth exploring the relationship between the role of the wave function within the coherentist proposal and the nomological account of the wave function (see Goldstein and Zanghì 2013; Solé and Hoefer 2019). According to the latter view, the wave function should not be regarded as a substantive physical entity. Instead, we should take the wave function in Bohmian mechanics as a nomological, law-like entity. The nomological account shares with the coherentist framework the claim that the wave function is not a material entity, since this status should be attributed only to the particles. However, there is a prima facie reason to claim that the coherentist account is not a version of the nomological interpretation, since the wave function has no governing role in the former account. That is, the wave function supervenes on the web of dependence, and does not govern the evolution of the particles per se. Thus, it seems to have a descriptive nature, not a nomological status. However, one could argue that having a governing role is not a necessary condition to be a law. Indeed, the Humean view about the nature of the laws denies that laws have the ability to govern, and considers laws of nature — very roughly_as salient regularities which can be tracked in the distribution of the fundamental intrinsic properties (in accordance with the thesis of Humean supervenience as mentioned above). If one adopts a very loose version of this account and regards laws as mere bookkeeping devices, supervening on the fundamental entities - thus, leaving aside the Humean ontology which underlies the account - then the wave function could be deemed as having a nomological status,

\footnotetext{
25 Remember that I am taking OSR, as well as coherentism, as metaphysical packages that concern the whole ontology of our world and not merely the metaphysical status of the wave function. Thus, when we confront coherentism with other accounts, we have to consider all the metaphysical implications of the theories.

26 To be fair, a structuralist Humean account has been proposed in the literature (see Lyre 2010; French 2014, ch. 9). This account adopts a version of structural realism very different from those discussed in this paper, which rejects the appeal to natural necessity in the world. Indeed, in this paper I assumed objective irreducible modality to be a necessary part of OSR. In any case, I believe that adopting a version of Humean structuralism in our context would be the same as adopting the Humean account of Bohmian mechanics as presented in this section, and thus I will not discuss that option separately.
} 
within the coherentist framework. ${ }^{27}$ In conclusion, if we assume that laws must govern then the coherentist proposal is not nomological, but, if we reject that assumption and we adopt a notion of laws of nature akin to the Humean one, then the wave function could be regarded as having a nomological status within the coherentist view. However, once again, the view does not collapse into the Humean account discussed before, due to the inherently structuralist/modal nature of the coherentist ontology, and neither it collapses into the standard nomological account discussed in the literature, which is introduced independently from any structuralist ontology (even though it can be deemed as a peculiar version of that view about the wave function).

Summing up, it can be stressed that the coherentist approach to the ontology of Bohmian mechanics should be distinguished from the other options available. On the one hand, it is distinct from the standard OSR interpretation as presented in the previous section; on the other hand, the coherentist view about Bohmian mechanics is clearly distinct from the Humean interpretation, and-even though it is compatible with a nomological understanding of the wave function- the coherentist account does not collapse on the standard nomological interpretation discussed in the literature, due to its peculiar structuralist ontology.

To conclude, I want to draw some final considerations about coherentism. Firstly, confronted to moderate OSR, coherentism is more elegant and more intuitive. Moderate OSR postulates objects, relations, and relations of ontological dependence between them. On the other hand, coherentism conveys a very similar picture just by relying on objects and relations of ontological dependence among them. ${ }^{28}$ Of course, whether this elegance in the formulation really mirrors a more parsimonious ontology depends on the issue I just mentioned a few paragraphs above.

Secondly, coherentism is in some sense a form of structuralism-it is simply that the structure at stake is constituted by webs of ontological dependence. Indeed, just like OSR, coherentism stresses the inter-dependent nature of the elements in the fundamental ontology. Thus, even though it can be considered an object-first ontology, it is very different from a standard object-oriented metaphysics, like the one advocated by Lewis (1986). In the light of this, I maintain that the coherentist interpretation of the wave function that I have set out in this section should not be considered as really an alternative to Lam and Esfeld's structuralist interpretation, but as a way to formulate their basic proposal more precisely. Leaving aside some details-such as the alleged 'concreteness' of the structure representing the wave function-the coherentist proposal is actually consistent with Lam and Esfeld's core intuitions.

Finally, and most importantly, when it comes to the ontology of Bohmian mechanics, coherentism seems to be the most straightforward account concerning the nature of the wave function. In fact, coherentism takes the Bohmian particles as the most fundamental entities of the theory. In this way, it vindicates Dürr's and Goldstein's quotes which I have reported in the second section-representing roughly the physicists' point of view on Bohmian mechanics-according to which "for Bohmian mechanics the

\footnotetext{
27 Esfeld and Lam (2010, sec. 8.5) argue that within OSR we can regard laws of nature as supervening on the modal structure of the world. The approach just introduced is very similar to this proposal.

28 One reason for which coherentism is more elegant than moderate OSR is that-due to the fact that coherentism postulates ontological dependences only between objects - the theory can be formulated using merely first-order logic, whereas moderate OSR requires second-order logic (see footnote 16).
} 
particles are primary, or primitive, while the wave function is secondary, or derivative". Indeed, within this interpretation the wave function emerges from the dependence relations between the particles. In this sense, the coherentist account is much less revisionary than the priority-based OSR one, which takes particles as derivatives.

\section{Conclusion}

Lam and Esfeld have proposed to combine Bohmian mechanics with OSR, to put forward a structuralist account about the ontology of Bohmian mechanics. In this paper, I have taken the first (and most natural) step towards the development of their structuralist approach. In fact, as I have pointed out, OSR is not simply a generic framework. Rather, it comprises a family of different positions. Therefore, my aim in this paper has been that of formulating the structuralist account in terms of eliminativist, priority-based and moderate OSR. Furthermore, I have confronted Lam and Esfeld's proposal with the framework of metaphysical coherentism. That is, I have sketched a coherentist-structuralist account of Bohmian mechanics.

While both the three OSRist accounts as well as coherentism are arguably equally viable alternatives to characterize the structuralist interpretation, at least prima facie the latter account seems to be in a better shape than the other ones. There is still work to be done to fully develop that proposal. However, my hope is that what I have shown so far could prove that this is a work worth pursuing.

Acknowledgements I am very grateful to James Ladyman, Claudio Calosi, Christian Wüthrich, David Schroeren, Kit Fine and two anonymous referees for their helpful and insightful comments on different versions and parts of this paper. I thank also the audience at the 2020 edition of the Australasian Postgraduate Philosophy Conference. This research has been supported and funded by SWW DTP (AHRC).

Funding Funding was provided by the Arts and Humanities Research Council.

Open Access This article is licensed under a Creative Commons Attribution 4.0 International License, which permits use, sharing, adaptation, distribution and reproduction in any medium or format, as long as you give appropriate credit to the original author(s) and the source, provide a link to the Creative Commons licence, and indicate if changes were made. The images or other third party material in this article are included in the article's Creative Commons licence, unless indicated otherwise in a credit line to the material. If material is not included in the article's Creative Commons licence and your intended use is not permitted by statutory regulation or exceeds the permitted use, you will need to obtain permission directly from the copyright holder. To view a copy of this licence, visit http://creativecommons.org/licenses/by/4.0/.

\section{References}

Albert, D. Z. (1996). Elementary quantum metaphysics. In J. T. Cushing, A. Fine, \& S. Goldstein (Eds.), Bohmian mechanics and quantum theory: An appraisal (pp. 277-284). Kluwer Academic Publishers.

Allori, V., Goldstein, S., Tumulka, R., \& Zanghi, N. (2008). On the Common Structure of Bohmian Mechanics and the Ghirardi-Rimini-Weber Theory Dedicated to GianCarlo Ghirardi on the occasion of his 70th birthday. British Journal for the Philosophy of Science, 59(3), 353-389.

Barnes, E. (2018). Symmetric dependence. In R. Bliss \& G. Priest (Eds.), Reality and its structure: Essays in fundamentality (pp. 50-69). Oxford University Press.

Belot, G. (2012). Quantum states for primitive ontologists: A case study. European Journal for Philosophy of Science, 2(1), 67-83. 
Calosi, C., \& Morganti, M. (2021). Interpreting quantum entanglement: steps towards coherentist quantum mechanics. The British Journal for the Philosophy of Science, 72:3, 865-891.

Chen, E. (2019). Realism about the wave function. Philosophy Compass, 14(7), 10.

Dewar, N. (2020). La bohume. Synthese, 197(10), 4207-4225.

Dürr, D., Goldstein, S., \& Zanghì, N. (2012). Quantum physics without quantum philosophy. Springer.

Esfeld, M. (2017). How to account for quantum non-locality: Ontic structural realism and the primitive ontology of quantum physics. Synthese, 194(7), 2329-2344.

Esfeld, M., \& Lam, V. (2008). Moderate structural realism about space-time. Synthese, 160(1), $27-46$.

Esfeld, M., \& Lam, V. (2010). Ontic structural realism as a metaphysics of objects. In Scientific structuralism (pp. 143-159). Springer.

Esfeld, M., Lazarovici, D., Hubert, M., \& Durr, D. (2013). The Ontology of Bohmian Mechanics. British Journal for the Philosophy of Science, 65(4), 773-796.

Fine, K. (1995). Ontological dependence. In Proceedings of the Aristotelian society (Vol. 95, pp. 269-290). Aristotelian Society, Wiley.

Fine, K. (2015). Unified foundations for essence and ground. Journal of the American Philosophical Association, 1(2), 296.

French, S. (2010). The interdependence of structure, objects and dependence. Synthese, 175(1), 89-109.

French, S. (2014). The structure of the world: Metaphysics and representation. Oxford University Press.

Goldstein, S. (2017). Bohmian mechanics. In E. Zalta (Ed.), Stanford encyclopedia of philosophy. Retrieved from https://plato.stanford.edu/archives/sum2017/entries/qm-bohm/.

Goldstein, S., \& Zanghì, N. (2013). Reality and the Role of the Wavefunction in Quantum Theory. In Ney, A. \& D. Z. Albert (Eds.), The Wave Function: Essays in the Metaphysics of Quantum Mechanics. Oxford University Press.

Ladyman, J. (2014). Structural Realism. The Stanford Encyclopedia of Philosophy (Winter 2020 Edition), Edward N. Zalta (ed.) https://plato.stanford.edu/archives/win2020/entries/structural-realism/.

Ladyman, J. (2015). Are there individuals in physics, and if so, what are they? In Guay, A., \& T. Pradeu (Eds.), Individuals across the sciences (pp. 193-206). Oxford University Press.

Ladyman, J. (2016). The foundations of structuralism and the metaphysics of relations. In A. Marmodoro, \& D. Yates (Eds.), The metaphysics of relations (pp. 177-197). Oxford University Press.

Ladyman, J., Spurrett, D., Ross, D., Collier, J., Spurrett, D., \& Collier, J. G. (2007). Every thing must go: Metaphysics naturalized. Oxford University Press.

Lam, V. (2015). Quantum structure and spacetime. In Metaphysics in contemporary physics (pp. 81-99). Brill Rodopi.

Lewis, D. (1986). Philosophical papers, Volume II. Oxford University Press.

Linnebo, O. (2008). Structuralism and the notion of dependence. The Philosophical Quarterly, 58(230), 59-79.

Lyre, H. (2010). Humean perspectives on structural realism. In The present situation in the philosophy of science (pp. 381-397). Springer.

McKenzie, K. (2017). Ontic structural realism. Philosophy. Compass, 12(4), 10.

McKenzie, K. (2020). Structuralism in the idiom of determination. The British Journal for the Philosophy of Science, 71(2), 497-522.

Miller, E. (2014). Quantum entanglement, Bohmian mechanics, and Humean supervenience. Australasian Journal of Philosophy, 92(3), 567-583.

Morganti, M. (2019). From ontic structural realism to metaphysical coherentism. European Journal for Philosophy of Science, 9(1), 7.

Morganti, M. (2020). Ontic structural realism and fundamentality. In Glick, D., G. Darby, \& A. Marmodoro (Eds.), The foundation of reality: Fundamentality, space, and time. Oxford University Press.

Ney, A. \& Albert, D. Z. (Eds.) (2013). The wave function: Essays in the metaphysics of quantum mechanics. Oxford University Press.

Suárez, M. (2015). Bohmian dispositions. Synthese, 192(10), 3203-3228.

Solé, A., \& Hoefer, C. (2019). The nomological interpretation of the wave function. In Philosophers look at quantum mechanics (pp. 119-138). Springer.

Tumulka, R. (2018). On Bohmian mechanics, particle creation, and relativistic space-time: Happy 100th Birthday, David Bohm! Entropy, 20(6), 462.

Publisher's Note Springer Nature remains neutral with regard to jurisdictional claims in published maps and institutional affiliations. 